\title{
BUCKLING BEHAVIOUR OF SINGLE-WALLED CARBON NANOTUBES UNDER AXIAL LOADING
}

\author{
Grzegorz Litak ${ }^{1}$ \\ 1 Lublin University of Technology, Faculty of Mechanical Engineering, Department of Automation, \\ Nadbystrzycka 36/268, 20-618 Lublin, Poland, e-mail: g.litak@pollub.pl
}

Received: 2016.12.15

Accepted: 2017.02.01

Published: 2017.03.01

\begin{abstract}
We investigate the behaviour of a single-walled Carbon Nanotube under axial compressive line load applied at both edges. The expected buckling response is studied by application of a molecular computation model. We formulate a global potential and search for its minimum to obtain the equilibrium configuration. The critical nanotube diameter, when local shell buckling occurs, is measured with two parameters: the value of compression loading and tube diameter.
\end{abstract}

Keywords: carbon nanotubes, buckling, stability.

\section{INTRODUCTION}

Carbon nanotubes (CNTs), after their discovery in 1991 by Iijima [6], attracted a lot interest from researchers working mainly in the new field of nanotechnology. Among unusual properties of these new structures there are mechanical properties $[3,8]$. For instance the Young's modulus of a carbon nanotube is expected to be several times that of the diamond $(\sim 1 \mathrm{TPa})$ reaching the extreme values compared to any other material. CNTs show various electrical properties as metallic conductors or semiconductors, depending on their physical properties [12].

Their unique physical properties have lead to various applications in different areas, such as scanning of chemicals content [7], nanoelectronics $[5,9]$ and biosensors [13, 2], nanoelectromechanical storage systems [4]. Nanotubes were applied as nanosensors and nanoactuators. Namely, vertically aligned single-walled carbon nanotubes were used in emission and vacuum micro-electronic devices, and as nano-, bio-sensors [13] and nanoactuators [5].

In this note, motivated by potential applications of these interesting materials [11], we examine a critical value of axial loading which leads to local buckling instability for a single walled carbon nanotube.

\section{MODEL ASSUMPTIONS}

In the first step we define interactions between carbon atoms forming a tube. They can be defined by a simplified inter-atomic phenomenological potential $V$ of Brenner-Teresoff type [1, 10]:

$$
V=\sum_{i, j(>i)} V_{R}\left(r_{i j}-\bar{B}_{i j} V_{A}\left(r_{i j}\right)\right.
$$

where $i$ and $j$ indicate the atomic sites, while $r_{i j}$ is the distance between $i$-th and $j$-th carbon atoms while $\overline{\mathrm{B}}_{\mathrm{ij}}=\left(\mathrm{B}_{\mathrm{ij}}+\mathrm{B}_{\mathrm{ji}}\right) / 2$. The related nearest neighbours configuration and the applied axial forces are presented in Figure 1. The system parameters are included in Table 1.

Figures $2 \mathrm{a}$ and $2 \mathrm{~b}$ show atomic bond configuration under loading for armchair carbon nanotubes of two different diameters. We show the deformation of a short piece of a carbon nanotube under uniaxial compression preserving the cylindrical symmetry. Such deformation is connected with small changes in nanotube geometry in both diameter and length. 
a)

b)

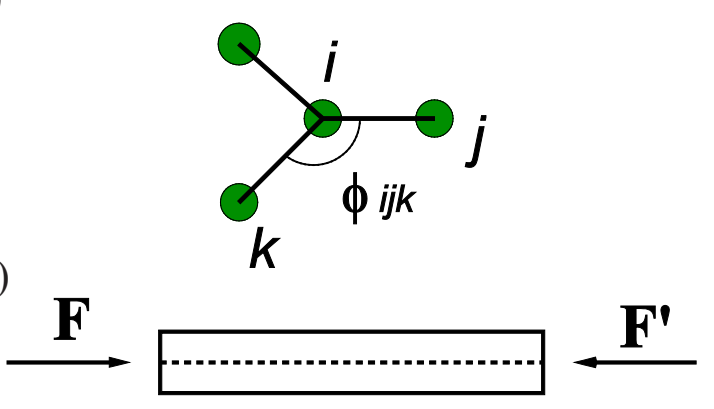

Fig. 1. Definition of nearest neighbour bonds angle $\varphi_{\mathrm{ijk}}(\mathrm{a})$, schematic plot of uniaxial compression $\left|\mathrm{F}^{\prime}\right|=|\mathrm{F}|=\mathrm{F}(\mathrm{b})$
Table 1. The table show a set of parameters used in calculations $[1,10]$

\begin{tabular}{|c|c|}
\hline Parameter & Value \\
\hline$R_{i j}^{(e)}$ & $1.39 \AA$ \\
\hline$D_{i j}^{(e)}$ & $6.0 \mathrm{eV}$ \\
\hline$\beta_{i j}$ & $2.1 \AA^{-1}$ \\
\hline $\mathrm{S}_{i j}$ & 1.22 \\
\hline$\delta_{i j}$ & 0.5 \\
\hline$\alpha$ & 0.0 \\
\hline $\mathrm{R}_{i j}^{(1)}$ & $1.7 \AA$ \\
\hline $\mathrm{R}_{i j}^{(2)}$ & $2.0 \AA$ \\
\hline$\alpha_{0}$ & 0.000200813 \\
\hline $\mathrm{c}_{0}$ & 330 \\
\hline $\mathrm{d}_{0}$ & 3.5 \\
\hline
\end{tabular}

\section{STABILITY ANALYSIS}

In the next step we analyse the atomic potential and stability of the deformed structures (Fig. 2). The repulsive and attractive parts of the potential: $V_{R}, V_{A}$ are defined as:

$$
\begin{gathered}
V_{R}\left(r_{i j}\right)=\frac{f_{i j}\left(r_{i j}\right) D_{i j}^{(e)}}{S_{i j}-1} \exp \left(-\sqrt{2 S_{i j}} \beta_{i j} \Delta r_{i j}\right) \\
V_{R}\left(r_{i j}\right)=\frac{f_{i j}\left(r_{i j}\right) D_{i j}^{(e)} S_{i j}}{S_{i j}-1} \exp \left(-\sqrt{2 S_{i j}} \beta_{i j} \Delta r_{i j}\right)
\end{gathered}
$$

where:

$$
\begin{gathered}
f_{i j}=\left\{\left[\begin{array}{c}
1, \quad r<R_{i j}^{(1)} \\
\left.1+\cos \left(\frac{\pi\left(r-R_{i j}^{(1)}\right)}{\left(R_{i j}^{(2)}-R_{i j}^{(1)}\right)}\right)\right] / 2, \quad R_{i j}^{(1)}<r<R_{i j}^{(2)} \\
0, \quad r>R_{i j}^{(2)}
\end{array}\right.\right. \\
B_{i j}=\left[\begin{array}{c}
1+\sum_{k(\neq i, j)} G_{i}\left(\phi_{i j k}\right) f_{i j k}\left(r_{i j}\right) \exp \left(\alpha_{i j k}\left[\Delta r_{i j}-\Delta r_{i k}\right]\right.
\end{array}\right]^{-\delta_{i j}} \\
G_{i}\left(\phi_{i j k}\right)=a_{0}\left\{1+c_{0}^{2} / d_{0}^{2}-c_{0}^{2} /\left[d_{0}^{2}+\left(1+\cos \phi_{i j k}\right)^{2}\right]\right\} \\
\Delta r_{i j}=r_{i j}-R_{i j}^{(e)}
\end{gathered}
$$

a)

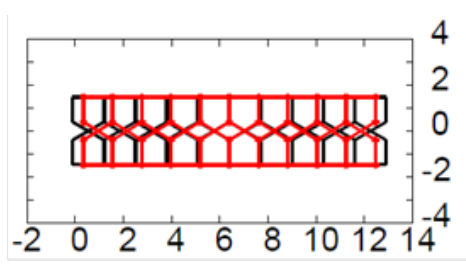

b)

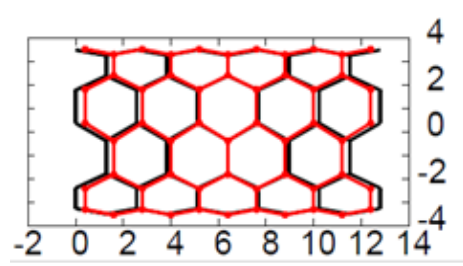

Fig. 2. Projections of $2 \times 2$ (a) and $5 \times 5$ (b) single walled carbon nanotubes structures (armchair) under an axially directed compression on their edges with a $\mathrm{F}=3 \mathrm{eV} / \AA$. Here, red lines correspond to loaded nanotube structures while black to unloaded ones (distances are expressed in carbon lattice spaces of the unloaded tube structure) 
Symbol $\phi_{\mathrm{ijk}}$ defines the angle between the lines, connecting $i$-th with $j$-th atom and $i$-th with $k$-th atom. $\mathrm{R}_{\mathrm{ij}}^{(\mathrm{e})}$ is the distance between atoms at equilibrium. All the potential parameters appearing in Equations 1-7 are given in Table 1, and were adjusted from Ref. [1].

To determine the stability of the given equilibrium structure under the loading we analyze the Jacobian matrix of the total potential $V_{T O T}\left(r_{1}\right.$, $\left.r_{2}, \ldots, r_{N}\right)$ composed of the inter-atomic potential $\mathrm{V}$ (Eq. 1) and the work of the external axial forces (Fig. 1b) $\mathbf{F}=-\mathbf{F}^{\prime}$.

To analyze the stability of atom configuration we used the condition that for a stable configuration of atoms eigenvalues of the Jacobian matrix calculated from $V_{T O T}$ with the following matrix elements:

$$
\frac{\partial^{2} V_{T O T}}{\partial \xi_{i} \partial \eta_{j}}
$$

where $\xi_{\mathrm{i}}, \eta_{\mathrm{j}}=\mathrm{x}_{1}, \mathrm{y}_{1}, \mathrm{z}_{1}, \mathrm{x}_{2}, \mathrm{y}_{2}, \mathrm{z}_{2}, \ldots, \mathrm{x}_{\mathrm{n}}, \mathrm{y}_{\mathrm{n}}, \mathrm{z}_{\mathrm{n}}$

are Cartesian coordinates of all $N$ atoms defined in the studied carbon nanotube. For a stable configuration all eigenvalues of the Jacobian matrix

a)

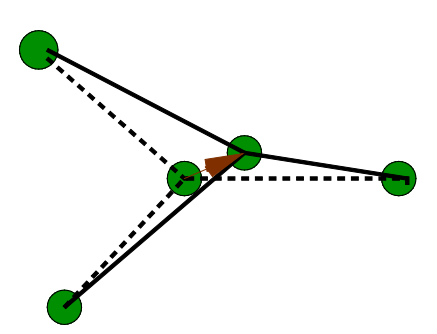

b)

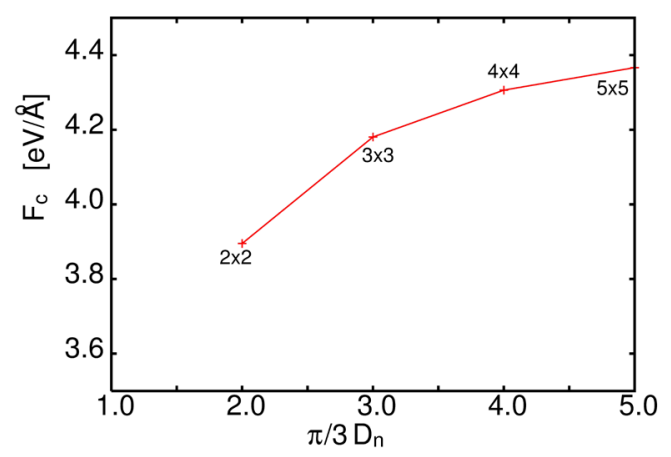

Fig. 3. Schematic plot of a single atom displacement (a) and critical values of the axial force versus the unloaded nanotube diameter $D_{n}=3 n / \pi$ (for the armchair structure of the size $n \times \bar{n}$ ) where the unit value is distance of carbon nearest atoms $\mathrm{R}_{\mathrm{ij}}{ }^{(\mathrm{e})}$. Note that the units of critical force $F_{c}$ is $1 \mathrm{eV} /{ }^{\circ} \mathrm{A}=1.602176462 \mathrm{nN}$ should be positive. In the present approximation we restricted the analysis to a single carbon atom displacement (Fig. 3a) where all surrounding atoms positions were fixed. The results of the critical force $F_{c}$ are shown in Figure 3b. Note that the critical force $F_{c}$, above which the system is unstable, is growing with the size of the unloaded nanotube diameter $D_{n}$. This represents a general trend expected to occur in any cylinder structure, which is usually more stable on buckling for larger $D_{n}$.

\section{CONCLUSIONS}

We investigated stability of a single-walled (armchair) Carbon Nanotube under axial compressive line loading applied at both of its edges. By analyzing the Jacobian matrix eigenvalues we successfully estimated the critical force leading to local bifurcations. To obtain more appropriate results of $F_{c}$ of a local buckling instability, one should go beyond a single atom displacement. The results of such an investigation are in preparation and they will reported in a separate paper.

\section{Acknowledgments}

The author would like to thank the Technical University of Vienna for hospitality and Professors H. Troger and A. Steindl for the support.

\section{REFERENCES}

1. Brenner D.W. Empirical potential for hydrocarbons for use in simulating the chemical vapor deposition of diamond films. Phys. Rev. B 42 (1990) 9458-9471.

2. Chowdhury R., Adhikari S., Mitchell J. Vibrating carbon nanotube based bio-sensors. Physica E 42 (2009) 104-109.

3. Coleman J.N., Khan U., Blau W.J., Gunko Y.K. Small but strong: A review of the mechanical properties of carbon nanotube-polymer composites. Carbon 44 (2006) 1624-1652.

4. Ding F., Lin Y., Krasnov P.O., Yacobsen B.I. Nanotube-derived carbon foam for hydrogen sorption. J. Chem. Phys. 127 (2007) 164-703.

5. Fennimore A.M., Yuzvinsky T.D., Han W.-Q., Fuhrer M.S., Cumings J., Zettl A. Rotational actuators based on carbon nanotubes. Nature 424 (2003) 408-410.

6. Iijima S. Helical micronanotubes of graphitic carbon. Nature 354 (1991) 56-58. 
7. Kumar O., Singh Y., Rao V.K., Vijayaraghavan R. Carbon nanotubes: detection of chemical and biological warfare agents. Defence Science Journal 58 (2008) 617-625.

8. Lu J.P. Elastic properties of single and multilayered nanotubes. J. Phys. Chem. Solids 58 (1997) 1649-1652.

9. Matsunaga N., Arie T., Akita S. A carbon nanotube field-effect transistor with a cantilevered carbon nanotube gate. Appl. Phys. Express 5 (2012) 65-101.

10. Proykova A., Iliev H. Simulated stress and stretch of SWCNT, in Proceedings of SIMS 2004 Copenhagen, Denmark, September 23-24, 2004, Editors: B. Elmegaard, J. Sporring, K. Erleben, K. Sorensen. Technical University of Denmark, Lyngby.

11. Schwarzbart M., Steindl A., H. Troger H. Buckling of carbon manotubes: A molecular static approach, PAMM 10 (2010) 27-30. 